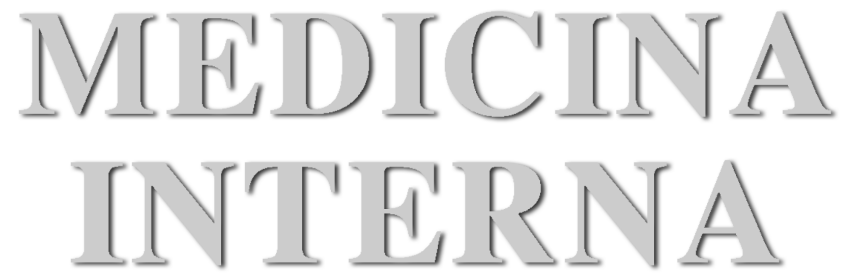

Fundada en 1983

Prof. J. de Portugal y Prof. M. Díaz Rubio

\title{
Cartas al Director
}

\section{Condensación pulmonar en adulto tras consulta con odontólogo}

\section{Sr. Director:}

La aspiración de un cuerpo extraño es una de las complicaciones más serias (pero afortunadamente poco frecuente) que puede ocurrir en el transcurso de un procedimiento dental. Debemos sospecharla cuando habiendo antecedente de dicho procedimiento el paciente presenta procesos respiratorios sobre todo si son larvados, aún en ausencia de clínica de aspiración durante el procedimiento dental.

Presentamos a continuación el caso de un varón de 60 años que consulta en el Servicio de Urgencias por fiebre con tiritona y tos escasa de diez días de evolución que no remiten con el tratamiento antitérmico pautado por su médico de atención primaria. En la exploración física cabe destacar temperatura axilar de $39,2^{\circ} \mathrm{C}$, saturación de $\mathrm{O}_{2}$ con pulsioxímetro digital del 93\%. A la auscultación pulmonar presenta hipoventilación en base pulmonar izquier$\mathrm{da}$, en el hemograma leucocitosis de $13.000 / \mathrm{ml}$ con neutrofilia y en la RX de tórax PA-L (Fig. 1) se objetiva cuerpo extraño metálico en lóbulo inferior izdo con condensación distal. Entonces reinterrogamos al paciente y recuerda que dos o tres días antes de comenzar con esta clínica había estado en el odontólogo para la colocación de una prótesis dental en la arcada superior, cayendo material durante la colocación. Dado que no presentó clínica evidente de broncoaspiración se pensó que había ido a vía digestiva.

Para la extracción del cuerpo extraño se realiza fibrobroncoscopia flexible bajo anestesia local y sedación lográndose extraer el mismo con una pinza ratón sin complicaciones. Se pautó tratamiento antibiótico con amoxicilina-clavulámico durante diez días estando el paciente afebril a las 24 horas de la realización de la fibrobroncoscopia y de comenzar el tratamiento antibiótico. Se realizó radiografía de control a las cuatro semanas con desaparición total de la imagen de condensación. El diagnóstico al alta médica fue de neumonía postobstructiva por cuerpo extraño metálico broncoaspirado durante la colocación de prótesis dental.

La aspiración de un cuerpo extraño en el transcurso de un procedimiento dental puede ser favorecida por la utilización de instrumental de pequeño tamaño por parte del odontólogo (1) y por la posición del paciente reclinado en supino $(1,2,4)$. Otros factores como la edad, la anestesia local y la disminución del nivel de con-

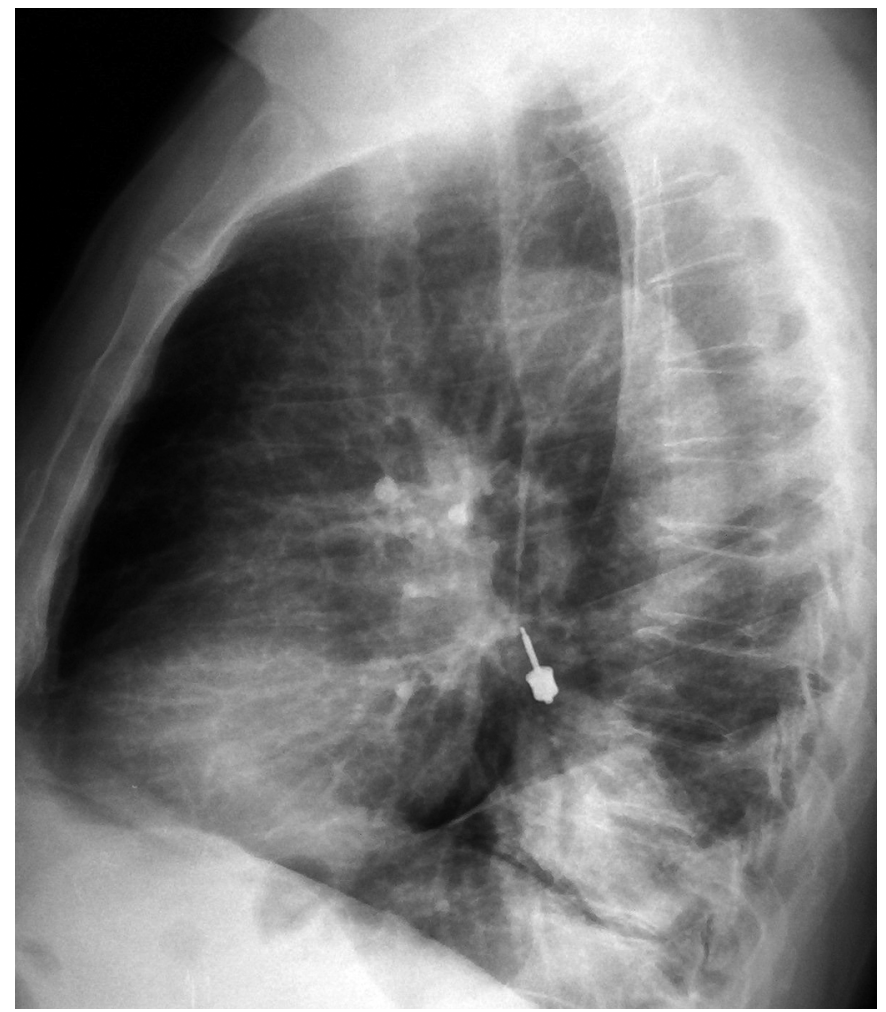

Fig. 1. Cuerpo extraño metálico en lóbulo inferior izquierdo con condensación distal afectando a segmento basal posterior. Radiografía de tórax lateral.

ciencia si se emplea cierta sedación con el paciente (1) pueden también facilitarla. Las consecuencias de la broncoaspiración pueden ser inmediatas como son la disnea, asfixia, asistolia, edema de laringe y neumotórax (4) y en el caso de pasar desapercibida pueden presentarse complicaciones tardías: neumonías de lenta resolución, abcesos pulmonares, bronquiectasias y hemoptisis $(1,3,4)$. Las complicaciones tardías son más frecuentes cuando son inhalados pequeños materiales inorgánicos porque en muchas ocasiones son asintomáticas las broncoaspiraciones, y la obstrucción total (distal al cuerpo aspirado) tarda en producirse (3). En adultos el lugar más 
común de impactación del cuerpo extraño es en el lóbulo inferior derecho $(3,4)$ por la propia anatomía bronquial. El material que más frecuentemente se aspira durante los procedimientos del odontólogo son las prótesis dentales (2). Otras son: dientes, gasas, destornilladores, agujas para la anestesia e incluso la propia barrera de goma que se emplea para evitar la aspiración (1,2). Existen distintos métodos barrera cuyo objetivo es el que no se produzca este evento, y que deberá utilizar el odontólogo según los procedimientos a realizar. Entre ellos está el dique de goma, que debe ser utilizada cuando se realizan endodoncias y restauraciones dentales, no pudiendo ser empleada en la cirugía oral ni en la colocación de prótesis dentales. En ese caso se podría colocar una gasa como barrera protectora (1). Si estas barreras no son efectivas y se produce una broncoaspiración, el tratamiento de elección en los adultos es una broncoscopia en un principio flexible (1-4). Si el diagnóstico se retrasa (como puede ocurrir sobre todo si los cuerpos extraños son radiotransparentes ya que la radiografía simple no permite su diagnóstico) pueden aparecer bronquiectasias con hemoptisis franca, requiriéndose lobectomía para su curación (3).

En nuestro caso el mayor de los problemas se derivó por la no sospecha de la broncoaspiración por parte del dentista, ya que aunque se sabía que habían caído dos piezas instrumentales el paciente no tuvo clínica alguna. Hubo la suerte de que se trató de un objeto radioopaco y se visializó en la radiografía de tórax realizada en nuestro Servicio de Urgencias, extrayéndose seguidamente y quedando el paciente asintomático 24 horas mas tarde.

\section{E. Guerra del Barrio, D. Ojer Tsakiridu ${ }^{1}$, J. Vallina García ${ }^{2}$}

Servicio de Medicina Interna. Hospital V. Álvarez Buylla. Mieres. ${ }^{\prime}$ Medicina de Familia $y^{2}$ Estomatología. Centro de Salud de la Felguera. Langreo. Asturias

1. Cameron SM, Whitlock WL, Tabor MS. Foreing body aspiration in dentistry: A review. J Am Dent Assoc 1996; 127 (8): 1224-9.

2. Sopena B, Garcia-Caballero L, Diz P, De la Fuente J, Fernandez, Diaz JA. Unsuspected foreign body aspiration. Quintessence Int 2003; 34 (10): 779-81.

3. Dikensoy O, Usalan C, Filiz A. Foreign body aspiration: clinical utility of flexible bronchoscopy. Postgrad Med J 2002; 78 (921): 399-403.

4. Limper AH, Prakash UB. Tracheobronchial foreign bodies in adults. Ann Intern Med 1990; 112 (8): 604-9.

\section{Enfermedad pulmonar por Mycobacterium scrofulaceum}

\section{Sr. Director:}

Las enfermedades producidas por las micobacterias ambientales (MA) se han convertido en los últimos 15 años en una afección relativamente frecuente, sobre todo condicionada por el sida (1). En EE.UU. las MA llegan a representar las tres cuartas partes de todos los aislamientos de micobacterias (2). En España, en un trabajo reciente, se ponía de manifiesto el incremento ocurrido en los últimos años en las infecciones causadas por las MA (3).

Mycobacterium scrofulaceum es una MA de crecimiento lento, del grupo de las escotocromógenas. Sus manifestaciones clínicas más frecuentes son la linfadenitis, que afecta fundamentalmente a los niños, y la enfermedad diseminada en inmunodeprimidos (4), siendo, por otro lado, raros, los casos publicados de enfermedad pulmonar por esta micobacteria $(5,6)$. Por este motivo, presentamos un caso de enfermedad pulmonar causado por Mycobacterium scrofulaceum y se revisan los criterios para su correcto diagnóstico.

Mujer de 87 años con mala situación basal, con antecedentes de tuberculosis pulmonar en la juventud, criterios clínicos de bronquitis crónica y dos ingresos en otro centro hospitalario por infección respiratoria en el último año. Un mes antes de su ingreso, inició un episodio de infección respiratoria y síndrome constitucional con pérdida de peso y astenia. Fue tratada ambulatoriamente con quinolonas sin presentar clara mejoría. En la exploración física sólo destacaba una discreta palidez y a la auscultación crepitantes diseminados y alguna sibilancia aislada. Aportaba una radiografía de tórax en la que se apreciaba paquipleuritis bilateral apical e imágenes fibrocavitarias de aspecto residual en el lóbulo superior derecho y en el lóbulo superior contralateral imagen infiltrativa con focos confluentes. En la analítica destacaba una hemoglobina de $10,9 \mathrm{~g} / \mathrm{dl}$ y una velocidad de sedimentación de 126. Se observaron BAAR en esputo y se realizaron hemocultivos que fueron estériles. Ante la sospecha de una reactivación de su tuberculosis antigua se instauró tratamiento con isoniacida, rifampicina y pirazinamida. Posteriormente el cultivo fue positivo e identificado como Mycobacterium scrofulaceum. Revisada la historia de los ingresos previos existían otros dos cultivos positivos pero considerados como colonizantes. El antibiograma demostró resistencia a fármacos de primera línea y a quinolonas, pero sensible a claritromicina, cicloserina, rifabutina y amikacina. Se diagnosticó de enfermedad pulmonar por Mycobacterium scrofulaceum aplicando los criterios de enfermedad por micobacterias ambientales establecidos por la American Thoracic Society (2) y se decidió tratar inicialmente con claritromicina $500 \mathrm{mg}$ cada 12 horas y rifabutina $150 \mathrm{mg}$. cada 24 horas. En el control analítico realizado a los 15 días del inicio se observó bicitopenia con 2800 leucocitos, 680 neutrófilos y 71.000 plaquetas, por lo que se decidió suspender temporalmente la medicación dado su estado general. Sin embargo, presentó un rápido deterioro clínico hasta su fallecimiento.

En nuestro país Mycobacterium scrofulaceum parece ser un agente etiológico poco frecuente. En un trabajo reciente representaba el 1,17\% de un total de 10.198 aislamientos de MNT (3) realizados en España durante el periodo 1993-1996. La enfermedad pulmonar parece presentarse de manera similar a la causada por otras MA (7). Los síntomas son variables y poco específicos, incluyen tos productiva crónica y disnea. Menos comunes son la fiebre y la hemoptisis y si el proceso está avanzado, pérdida de peso. La evaluación de estos síntomas suele ser complicada ya que suele coincidir con otras patologías como enfermedad pulmonar obstructiva crónica, tabaquismo, bronquiectasias, cicatrices de una tuberculosis pasada o silicosis (2). La cuestión sobre si la enfermedad por MA difiere radiologicamente de la enfermedad tuberculosa ha sido tratada en varias ocasiones con resultados dispares $(8,9)$. Sin embargo algunas características, concretamente, las cavidades de pared fina, la enfermedad bilateral, la intensa reacción pleural local e incluso un nódulo pulmonar solitario han sido citadas como más sugestivas de enfermedad por MA que por $M$. tuberculosis $(2,10)$.

Para el diagnóstico de enfermedad pulmonar producida por micobacterias ambientales, se recomienda utilizar los criterios de la American Thoracic Society (2) expuestos en la tabla I.

Debido a la escasez de ensayos terapéuticos no existen claras recomendaciones sobre cuál debe ser el tratamiento y la duración del mismo. La American Thoracic Society recomienda no utilizar ni pirazinamida ni isoniacida en el tratamiento de las MA de crecimiento lento, pudiendo ser útiles otros fármacos como la rifampicina, claritromicina, etambutol, quinolonas, rifabutina o ciprofloxacino, en cualquier caso siempre dependiendo de las pruebas del antibiograma. Si se produce respuesta clínica y bacteriológica al tratamiento, éste debe mantenerse al menos durante 18 a 24 meses (2). 
TABLA I

CRITERIOS DIAGNÓSTICOS ENFERMEDAD PULMONAR POR MICOBACTERIAS AMBIENTALES

\begin{tabular}{ccc}
\hline Clínicos & $\begin{array}{c}\text { Síntomas y signos compatibles (tos, fiebre, pérdida de peso, } \\
\text { hemoptisis o disnea) con deterioro del estado clínico. } \\
\text { Exclusión de otras enfermedades o tratamientos de otras } \\
\text { enfermedades que pudieran producir un deterioro clínico. }\end{array}$ \\
\hline \multirow{3}{*}{ Radiológicos } & En Radiografía Tórax: & En TAC de Tórax: \\
& -Infiltrados con o sin nódulos & - Múltiples nódulos de \\
& -Cavitación & pequeño tamaño \\
& -Nódulos únicos o múltiples & - Bronquiectasias mul- \\
& & tifocales con o sin \\
& & pequeños nódulos \\
& & pulmonares \\
\hline
\end{tabular}

Bacteriológicos \& Siempre que se puedan obtener, al menos, tres muestras de esputo o lavado broncoalveolar (LBA) en un año: tres cultivos positivos con baciloscopias negativas, o dos cultivos positivos y una baciloscopia positiva.

Ante la incapacidad de obtener esputos, un LBA con cultivo positivo o cultivo positivo con una baciloscopia positiva.

Biopsia:

- Cualquier crecimiento en los cultivos de muestras obtenidas a partir de biopsias broncopulmonares

- Granulomas y/o visión de bacilos ácido-alcohol resistentes en una biopsia pulmonar con uno 0 más cultivos positivos de esputo o LBA.

- Cualquier crecimiento obtenido de muestras extrapulmonares estériles.

En pacientes inmunodeprimidos se aceptan los mismos criterios, con la excepción de que se considera diagnóstico un cultivo positivo con un crecimiento de 1+ o más.

\section{R. Rodríguez Barrientos, J. L. Vidal Pérez'1, A. Noguerado Asensio $^{1}$}

Centro de Salud Chopera. Alcobendas. ${ }^{\text {SServicio de Medicina }}$ Interna V. Unidad de Aislamiento. Hospital Universitario Gregorio Marañón-Cantoblanco. Madrid

1. Medina Cruz MV, Sauret Valet J, Caminero Luna LA. Enfermedades producidas por micobacterias ambientales. Med Clin (Barc) 1999; 113: 621-630.

2. American Thoracic Society. Diagnosis and Treatment of Disease Caused by Nontuberculous Mycobacteria. Am J Crit Care Med 1997; 156; S1-S25.

3. Martín Casabona N, Rosselló Urgell J. Grupo de Estudio sobre Micobacterias Ambientales. Med Clin (Barc) 2000; 115: 663-670.

4. Núñez Fernández MJ, Ojea de Castron R, Anibarro Garcia L, Pascual Durán A. Infección diseminada por Mycobacterium scrofulaceum en un paciente infectado por el VIH. An Med Interna (Madrid) 2002; 19: 6364

5. Wolinsky E. Mycobacterial diseases other than tuberculosis. Clin Infect Dis 1992; 15: 1-12.

6. Sanders JW, Walsh AD, Snider RL, Sahn EE. Disseminated Mycobacterium scrofulaceum infection: a potentially treatable complicatión of AIDS. Clin Infect Dis 1995; 20: 549-56

7. Hautmann G, Lotti T. Diseases caused by Mycobacterium scrofulaceum. Clin Dermatol 1995; 13: 277-280.
8. Evans SA, Crisp AJ, Hubbard RB, Colville A, Evans AJ. Pulmonary Mycobacterium kansasii infection: comparision of radilogical appearances with pulmonary tuberculosis. Thorax 1996; 51: 1243-1247.

9. Woodring $\mathrm{J} \mathrm{H}$, Vandiviere H M. Pulmonary disease caused by nontuberculous mycobacteria. J Thoracic Imaging 1990; 5: 64-76.

10. Corbett EL, Hay M, Churchyard GJ, Herselman P. Mycobacterium kansasii and M scrofulaceum isolates from HIV-negative South African gold miners: incidence, clinical significance and radiology. T J Tuberc Lung Dis 1999; 3: 501-507.

\section{Hiperaldosteronismo e hiperparatiroidismo: ¿causalidad o casualidad?}

\section{Sr. Director:}

En los últimos años se ha comunicado la asociación entre hiperparatiroidismo primario e hiperaldosteronismo primario, no llegándose a una conclusión final sobre la relación entre ambas entidades.

Presentamos el caso de una mujer de 50 años con antecedentes de cólicos nefríticos de repetición, pancreatitis aguda e hipertensión de 15 años de evolución en tratamiento con beta-bloqueantes, IECAS y diuréticos, remitida para estudio por mal control de cifras tensionales. Durante el estudio se objetivó: calcio 11,9 $\mathrm{mg} / \mathrm{dl}(8-10,3)$, fósforo $2,36 \mathrm{mg} / \mathrm{dl}(2,5-4,5)$, PTH molecular intacta 246 , calciuria 24 h. $484 \mathrm{mcg} / 24 \mathrm{~h}$, fosfaturia 1641,6 $\mathrm{mcg} / 24 \mathrm{~h}$. y gammagrafía de paratiroides con presencia de adenoma siendo diagnosticada de hiperparatiroidismo primaria. Por otro lado se obtuvieron los siguientes resultados: Aldosterona $1.249 \mathrm{pg} / \mathrm{ml} / \mathrm{h}(40-300 \mathrm{pg} / \mathrm{ml} / \mathrm{h})$, Actividad renina plasmática $0,5 \mathrm{ng} / \mathrm{ml} / \mathrm{h}(1,40 \pm 0,92 \mathrm{ng} / \mathrm{ml} / \mathrm{h})$, gammagrafia de suprarenales con I-Colestorol compatible con hiperplasia suprarrenal bilateral, TC de suprarrenales sin presencia de adenoma, y renograma isotópico sin compromiso vasculorenal.

La asociación de hiperparatiroidismo e hiperaldosteronismo primario es excepcional (1-3). De la posible relación entre ambas patologías han surgido dos teorías enfrentadas. La primera; propuesta entre otros por Barkan A, y cols. (4) promueve que el hiperparatiroidismo es el responsable del hiperaldosteronismo. En tal sentido observaron que la extirpación del adenoma paratiroideo producía una normalización de las cifras tensionales, las alteraciones electrolíticas y de la actividad de la renina plasmática; sugiriendo que el responsable de la hipertensión es la acción directa del calcio iónico sobre la contracción de la pared arteriolar. La segunda teoría, fomentada en otros por Dorotea y cols. (5) opina que el hiperaldosteronismo es el responsable del hiperparatirodismo; ya que el hiperaldosteronismo primario cursa típicamente con alcalosis metabólica, la cual determinaría una reducción del calcio iónico; con el consiguiente estímulo de PTH mediante mecanismo de feedback (6). Los autores objetivaron la normalización de las cifras tensionales tras instaurar tratamiento con Espironolactona (7).

En relación con nuestra paciente el antecedente de cifras de fosfatemia bajas, historia de cólicos nefríticos de repetición y pancreatitis nos podría hacer pensar erróneamente que fue el hiperparatiroidismo el responsable del hiperaldosteronismo; pero por el contrario ha sido el tratamiento con espironolactona el que ha conseguido controlar las cifras tensionales hasta día de hoy.

Atribuimos la comunicación de casos coincidentes a una mayor sensibilidad de las técnicas diagnósticas que desenmascaran numerosa patología silente, y a la elevada incidencia de ambas patologías por separado en la población general (8-11). 
Por ello creemos que con los datos existentes hasta el momento no cabe concluir razonablemente una asociación causal entre ambas entidades.

\section{Teijo Núñez, A. Martínez González, E. Fernández Pérez, S. Pérez Andrada, M. Muñoz Rodríguez}

Servicio de Medicina Interna I. Hospital de León. León

1. Honda M, Tsukada T, Horiuchi T, Tanaka R, Yamaguchi K, Obara T, et al. Primary hyperparathyroidism associatiated with aldosterone-producing adrenocortical adenoma and breast cancer: relation to MEN1 gene. Intern Med 2004; 43: 310-314.

2. Makino S, Oda S, Saka T, Yasukawa M, Komatsu F, Sasano H. A case of aldosterone-producing adrenocortical adenoma associated with preclinical Cushing's syndrome and hypersecretion of parathyroid hormone. Endocr J 2001; 48: 103-111.

3. Estopiñán V, Fortea Gimeno L. Hiperparatiroidismo primaria e hiperaldosteronismo primaria: una rara asociación. Med Clin (Barc) 1992; 98: 117.

4. Barkan A, Marilus R, Winkelsberg G, Yeshurun D, Blum I. Primary hyperaldosteronism: possible cause of primary hyperaldosteronism in a 60 -year- old woman. J Clin Endocrinol Metab 1980; 51: 144-147.

5. Dorotea E. Hellman, Mark Kartchner, Norman Komar, Darrell Mayes, Michael Pitt. Hyperaldosteronism, Hyperparathyroidism, Medullary Sponge Kidneys , and hypertension. JAMA 1980; 244: 1351-1353.

6. Davies JS, Aguirre G, Cassidy DM, Griffiths BE. Latent tetany associated with the hypokalaemia of Conn's syndrome. Int J Clin Pract 1998; 52: 347-348

7. Rossi E, Sani C, Perazzoli F, Casoli MC, Negro A, Dotti C. Alterations of calcium metabolism and of parathyroid function in primary aldosteronism, and their reversal by spironolactone or by surgical removal of aldosterone-producing adenomas. Am J Hypertens 1995; 8: 884-893.

8. Conroy S, Moulias S, Wassif WS. Primary hyperparathyroidism in the older person. Age Ageing 2003; 32: 571-578.

9. Tomasella G. Diagnostic imaging in primary hyperparathyroidism. Radiological techniques: US-CAT-MR. Minerva Endocrinol 2001; 26: 3-12.

10. Nadar S, Lip GY, Beevers DG. Primary hyperaldosteronism. Ann Clin Biochem 2003; 40: 439-452.

11. Quinkler M, Lepenies J, Diederich S. Primary hyperaldosteronism. Exp Clin Endocrinol Diabetes 2002; 110: 263-271.

\section{Aneurisma de arteria pulmonar izquierda}

\section{Sr. Director:}

El aneurisma de la arteria pulmonar es una entidad muy poco frecuente, existiendo pocos casos comunicados a lo largo de la literatura médica $(1,2)$. Presentamos a continuación nuestra breve experiencia en este tema.

Paciente de 72 años de edad sin antecedentes médicos previos que encontrándose previamente bien, es ingresado en el Servicio de Medicina Intensiva ante súbito deterioro neurológico, en situación de coma profundo, con hemiplejía derecha y puntuación de Glasgow de 5 por lo que precisa intubación orotraqueal y ventilación mecánica.

Se realiza TAC cerebral urgente apreciándose un ictus silviano izquierdo extenso. En la Rx tórax destaca la existencia de un hilio pulmonar izquierdo prominente. Ante este hallazgo, y bajo sospecha de un proceso tumoral en dicha localización, se procede a realización de TAC torácica con contraste, donde se aprecia un aneurisma de la arteria pulmonar izquierda (Fig. 1) con

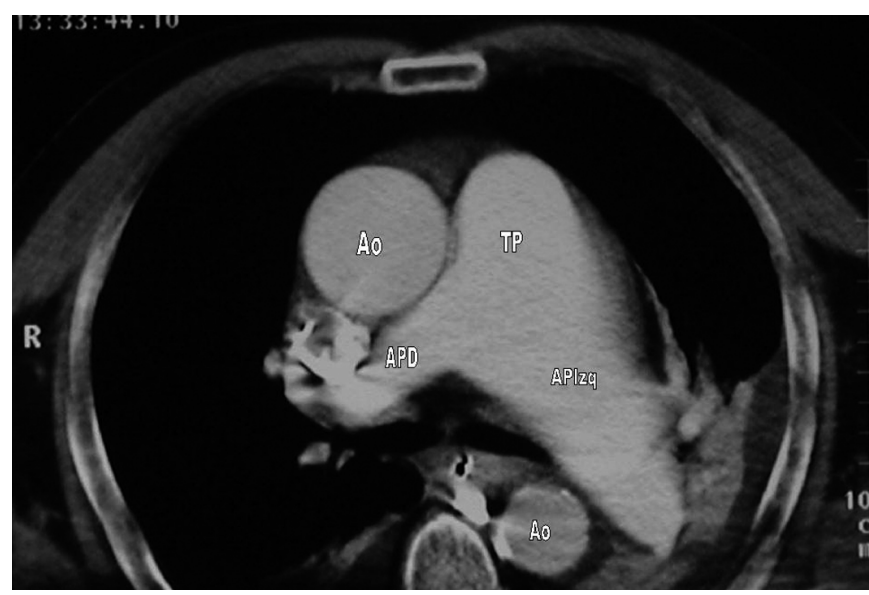

Fig. 1.

cavidades cardiacas de tamaño normal tanto en la TAC como en el ecocardiograma transtorácico realizado posteriormente, en el que tampoco se apreciaron signos de hipertensión pulmonar ni flujos sugerentes de conducto arterioso persistente ni otro tipo de shunt.

Bajo la forma de presentación neurológica llegamos al singular diagnóstico de aneurisma de la arteria pulmonar. Previamente a su ingreso el paciente no contaba con historia clínica de alteraciones cardiopulmonares y, durante el mismo, la exploración física no reveló ningún signo patológico que hiciera sospechar la existencia de valvulopatía mitral, signos de hipertensión pulmonar o presencia de patología respiratoria. Resulta sorprendente no apreciar en la ecocardiografía realizada signos de hipertensión pulmonar ni alteraciones valvulares que justificaran la existencia de dicho aneurisma, teniendo además en cuenta el tamaño del mismo.

\section{B. Obón Azuara, M. C. Ferrer Gracia ${ }^{1}$, B. Daga Calejero ${ }^{1}$, I.} Gutiérrez Cía

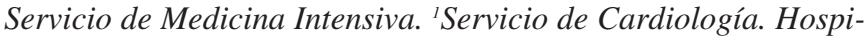
tal Clínico Universitario. Zaragoza

1. Hunter Cherwek M, Stan Amundson DN. Pulmonary-artery aneurism. Engl J Med 2003; 384: 1.

2. Sommez B, Tansal S, Unal M, Korkuy A, Yagan N, Demirsoy E, Arbatli H, Aytekin VJ. A left pulmonary artery aneurysm secondary to pulmonary hypertension. Cardiovasc Surg 2001; 42: 629-32.

\section{Artritis séptica por Neisseria meningitidis}

\section{Sr. Director:}

Entre los gérmenes más comunes que se aislan en las artritis sépticas no gonocócicas (ASNG) se encuentran, S. aureus, Streptococcus spp, bacilos gramnegativos y anaerobios, siendo el patógeno más frecuente $S$. aureus $(70 \%)$, también es el más frecuente en las artritis sépticas cuando afecta a los ADVP, SIDA, en mayores de 60 años y cuando la enfermedad de base es la artritis reumatoide (AR) (1) 
A continuación describimos un caso de monoartritis aguda poco común producida por Neisseria meningitidis en una paciente con AR.

Mujer de 68 años de edad, ingresa en el Servicio de Reumatología, por artritis séptica de rodilla derecha. Destaca de sus antecedentes personales, estar afecta de AR seropositiva de 25 años de evolución, tratada con deflazacort, celecoxib, omeprazol y suplemento de calcio. La paciente presenta buen estado general. Exploración neurológica normal sin rigidez de nuca ni signos meníngeos, exploración torácica y abdominal dentro de la normalidad. Sin fiebre y sin traumatismo previo, no adenopatías, ni exantema cutáneo, a nivel del aparato locomotor en miembros superiores presenta signos residuales de AR, en miembros inferiores, en rodilla derecha son evidentes los signos clásicos de inflamación, tumefacción, enrojecimiento, dolor e impotencia funcional. Entre las pruebas complementarias solicitadas: hemograma: HM: 4.69 millones $/ \mathrm{mm}^{3}$; HB: 9,2 g/dl; Hc: 32,6\% ; VCM: $69,6 \mathrm{fl}$; leucocitos: 9,140 x $103 / \mathrm{mm}^{3}$ (74,6\% PMN, 15,1\% Linfocitos, 7,2\% monocitos); plaquetas: 308 x 103/mm³. VSG: $90 \mathrm{~mm} / \mathrm{h}$. La bioquímica, estudio de hormonas tiroideas y de coagulación eran normales. PCR: 54,7 mgr. FR: 216. En la radiografía de rodillas se observa imagen lítica subcondral en platillo tibial externo en la rodilla derecha. Se obtiene líquido articular tras realizar artrocentesis, de aspecto inflamatorio, con 70.400 células $/ \mathrm{mm}^{3}$ (PMN: 94\%); Gluc: $38 \mathrm{mg} / \mathrm{dl}$; Prot: 3.900 mg/dl; LDH: 6.238. Las baciloscopias del líquido articular fueron negativas, en el cultivo del líquido se aisla $N$. meningitidis serogrupo B subtipo 4: P 1,15 los hemocultivos, el cultivo de exudado vaginal y faríngeo fueron negativos, VIH negativo. Mejorando clínica y analíticamente de la articulación con cloxacilina y ceftriaxona via parenteral, artrocentesis seriadas y lavado articular, obteniéndose cultivos negativos se procede al alta con antibioterapia oral durante diez días, cefuroxima-axetilo $500 \mathrm{mg} 1 \mathrm{comp} / 12 \mathrm{~h}$, cloxacilina 500 mg 1 comp/12h.

Discusión: Los pacientes con AR tienen un riesgo especialmente elevado de artritis bacteriana (prevalencia del 0,3 al 3,0\%, incidencia anual $0,5 \%$ ) con mal pronóstico funcional y tasa de mortalidad alta ( $25 \%$ frente al $9 \%$ en pacientes sin AR) debido a la presencia de factores de riesgo adicionales, como tener una enfermedad crónica de base o tratamiento con corticoides (1).

La artritis séptica por $N$. meningitidis se encuentra entre 1.6$16 \%$ como manifestación de infección meningocócica, se describe en el $50 \%$ de los niños y en el $11 \%$ de los adultos. En ausencia de meningitis o meningococemia es poco frecuente, aislándose en líquido articular en el 1\% de las artritis sépticas (2).

Shaad postula cuatro mecanismos patogénicos diferentes que producen la artritis meningocócica: Invasión bacteriana directa del líquido sinovial, reacción de hipersensibilidad por depósitos de inmunocomplejos, hemartrosis por la coagulación intravascular diseminada que se produce en la meningococemia fulminante $\mathrm{y}$ por último iatrogénica (2-4).

Fam y cols. clasifica las artritis en cuatro patrones según su presentación clínica: artritis en el seno de una enfermedad meningocócica aguda que a su vez se dividen en dos subtipos, la de comienzo temprano que ocurre en los primeros 5 días, de forma aguda, con exantema cutánea en el $96 \%$, meningitis en el $26 \%$ de los casos, los hemocultivos suelen ser positivos, esta artritis suele ser de corta duración y de pronta respuesta al tratamiento y relacionada directamente con la bacteriemia, su clínica puede ser indistinguible del síndrome artritis-dermatitis causada por una gonococemia. El otro subtipo es la artritis de comienzo tardío, se presenta de forma subaguda, mono u oligoarticular que ocurre después de los 5 primeros días de la infección meningocócica, los hemocultivos suelen ser negativos, el líquido articular es positivo entre $12-50 \%$ de los casos, es de resolución lenta, entre 1 a 4 semanas, se considera que está mediada por el sistema inmune. La artritis en la meningococemia crónica es infrecuente, presenta bacteriemia persistente y exantema cutáneo respondiendo bien al tratamiento antibiótico. Como caso primario es una forma muy rara de enfermedad meningocócica, afecta princi- palmente a niños, de sexo masculino $(80 \%)$ y es raro describirlo en adultos. Con afectación de vías respiratorias superiores en el $50 \%$ de los casos, $30 \%$ presenta exantema maculopapular, es poliarticular en un 30\% y monoarticular en el 50\%. Afecta a grandes articulaciones, la rodilla en el $60 \%$ de los casos. Se aísla del líquido articular en el 90\%, hemocultivos en el $40 \%$ y de faringe en el $30 \%$ de los casos. La respuesta al tratamiento es lenta pero de buen pronóstico. El serogrupo C es responsable del 36\%, el B del 30 y W135 13\%, también se han descrito casos con serogrupo A $(3,4)$.

El caso descrito entra a formar parte de este último grupo, sin exantema, los cultivos faríngeo y vaginal son negativos, añadiendo a la rareza del caso el ser una mujer adulta.

Teniendo en cuenta que la inmunodepresión favorece la infección por gérmenes capsulados, se han descrito casos en pacientes con déficit de complemento, lupus eritematoso sistémico, SIDA, mieloma múltiple, macroglobulinemia de Waldeström y leucemia linfocítica crónica pero no hemos encontrado en nuestra revisión de la literatura casos asociados a artritis reumatoide (5-8).

La alta prevalencia de artritis primaria meningocócica en niños coincide con el aumento en general de la infección por meningococo relacionado con un título bajo de anticuerpos contra $N$. meningitidis observado durante los primeros 2 años de vida. Esta relativa deficiencia del sistema inmune favorece la artritis primaria meningocócica en la infancia (9).

Artritis séptica ocurre como complicación de meningitis por meningococo más a menudo en el curso agudo de meningitis o meningococemia y menos en la crónica y como caso primario de artritis (10).

La forma de presentación de las ASNG es generalmente aguda, monoarticular y de peor pronóstico que las artritis gonocócicas (AG). El pronóstico de las artritis producida por $N$. meningitidis es más favorable que el de aquellos casos en que se aislan otros microorganismos habituales en este tipo de infecciones por ejemplo, S. aureus (10).

En caso de artritis sépticas de causa desconocida se debe considerar a $N$. meningitis como posible agente etiológico en pacientes adultos, e investigar la posibilidad de una inmunodepresión de base.

\section{R. Tejero García, J. Muñoz Molinero, M. J. Lacasa Díaz, R. Gordillo Urbano, F. Franco Álvarez de Luna, F. Rodrí- guez López ${ }^{1}$}

Servicio de Microbiología y Parasitología Clínica y ${ }^{\text {SServicio de }}$ Reumatología. Hospital Universitario Reina Sofía. Córdoba

1. Manual Merck. Infecciones de huesos y articulaciones (54). Merck Sharp \& Dohme de España S. A. 2000.

2. Schaad UB. Artritis in disease due to Neisseria meningitis. Rev Infec Dis 1980; 2: 880-888.

3. Wells M. Primary Meningococcal Artritis: Case Report and Review of the Literature. Military Medicine. 1997; 162: 769-72.

4. Hernández A, Echániz A, Freire M, et al. Artritis meningocócica primaria: dos casos en adultos. Enferm Infecc Microbiol Clin 1999; 5: 249-50.

5. Singwe-Ngandeu M, Buchs N, Rohner P, et al. Waldenström's Disease Complilcated by Recurrent Meningococcal Artritis. J Clin Microbiol 2001; 39: 3013-4.

6. Miller MI, Hoppmann RA, Pisko EJ. Multiple Myeloma Presenting with Primary Meningococcal Artritis. Am J Med 1987; 82: 1257-8.

7. Harcup C, Wing EJ, Schneider S, et al. Primary meningococcal arthritis and pseudogout in an elderly woman. Artritis Rheum 1983; 11: 140911.

8. Travis S, Wright EP, Innes EH. Primary meningococcal arthritis. J Infect 1989; 19: 79-80

9. Bongers V, Blaauw A, Bijlsma J, Hoepelman A. Primary oligoarthritis in a parent of a child with meningococcal group B sepsis and meningitis. Netherlands J Med 1998; 52: 193-6.

10. Apicella MA. Neisseria meningitidis. Mandell, Douglas and Bennett's principles and practice of infectious diseases. $5^{\mathrm{a}}$ ed. Churchill Livingstone, 2000; p. 1601-12. 


\section{Secreción inadecuada de hormona antidiurética secundaria a paroxetina}

\section{Sr. Director:}

El síndrome de secreción inadecuada de hormona antidiurética (SIADH) se ha descrito asociado a alteraciones del sistema nervioso central, hipotiroidismo, insuficiencia adrenal, neoplasias, enfermedades pulmonares, intervenciones quirúrgicas y varios fármacos psicoactivos (risperidona, amitriptilina, desipramina, lofepramina, carbamacepina...). Recientemente se han comunicado casos de SIADH en relación con inhibidores selectivos de recaptación de serotonina (ISRS), fundamentalmente con fluoxetina y de forma excepcional con fluvoxamina, sertralina y paroxetina. Describimos el caso de una mujer diagnosticada de depresión que presentó SIADH reversible secundario a tratamiento con paroxetina.

Se trata de una mujer de 82 años que ingresa por cuadro de deterioro de su estado general.

En sus antecedentes personales destacaba el diagnóstico de diabetes mellitus no insulín dependiente, vasculopatía periférica e insomnio y estaba en tratamiento con glimepirida $2 \mathrm{mg}$ a la comida, acarbosa $100 \mathrm{mg} / 8$ horas, zopiclona 7,5 mg/noche, pentoxifilina $400 \mathrm{mg} / 12$ horas y ticlopidina $250 \mathrm{mg} / 12$ horas; este tratamiento no había sufrido modificaciones en los últimos dos años. La paciente 15 días antes del ingreso había sido diagnosticada de síndrome depresivo e iniciado tratamiento con paroxetina 20 mg/día; días después inicia cuadro de intolerancia alimenticia con nauseas, astenia, malestar general y lentitud en el lenguaje con progresiva tendencia a la somnolencia. En la exploración presentaba tensión arterial de 110/60 $\mathrm{mmHg}$, frecuencia cardiaca de 70 latidos / min., temperatura $36^{\circ} \mathrm{C}$, no había signos de deshidratación, soplo sistólico de II/VI, lenguaje lento con tendencia al sueño, sin otros datos relevantes en la exploración física.

En la analítica destacaba: urea en plasma de $50 \mathrm{mg} / \mathrm{dl}$, creatinina $0,9 \mathrm{mg} / \mathrm{dl}$, sodio $119 \mathrm{mEq} / \mathrm{l}$, potasio $3,3 \mathrm{mEq} / \mathrm{l}$ y la osmolaridad en plasma $254 \mathrm{mmol} / \mathrm{Kg} \mathrm{H}_{2} \mathrm{O}$. En la orina el sedimento era normal, no había proteinuria, el sodio era de 54 $\mathrm{mEq} / \mathrm{l}$, potasio $7 \mathrm{mEq} / \mathrm{l}$ y la osmolaridad urinaria de 950 $\mathrm{mmol} / \mathrm{Kg} \mathrm{H}_{2} \mathrm{O}$. Se realizó analítica de hormonas tiroideas y cortisol basal que fueron normales. La radiografía de tórax no mostraba alteraciones de interés y en el TAC cerebral realizado, salvo atrofia cortical, no se detectaron anomalías. La paroxetina fue suspendida y se realizó restricción hídrica (1000 $\mathrm{ml} /$ día). La paciente mejoró clínicamente en los días siguientes, siendo dada de alta a los 18 días con una creatinina plasmática de $1 \mathrm{mg} / \mathrm{dl}$, urea $45 \mathrm{mg} / \mathrm{dl}$, sodio $142 \mathrm{mEq} / 1$ y potasio $4,4 \mathrm{mEq} / \mathrm{l}$. Pensamos que en el caso de nuestra paciente la causa de SIADH fue el tratamiento con paroxetina iniciado 15 días antes, dada la asociación temporal, la ausencia de otras posibles causas del síndrome y la remisión del cuadro con la suspensión del fármaco y la restricción hídrica.

El SIADH es un cuadro habitualmente reversible diagnosticado y tratado a tiempo, pero en caso contrario puede conducir al coma y a la muerte; sus manifestaciones clínicas características incluyen letargia, anorexia, nauseas, vómitos, calambres musculares, astenia, confusión y convulsiones. Los criterios aceptados como diagnósticos de SIADH y que cumplía nuestra paciente son: disminución de la osmolaridad plasmática $<275$ $\mathrm{mOsm} / \mathrm{kg} \mathrm{H}_{2} \mathrm{O}$ o Na plasmático $<135 \mathrm{mEq} / \mathrm{l}$, concentración urinaria inapropiada (osmolaridad urinaria $>100 \mathrm{mOsm} / \mathrm{kg} \mathrm{H}_{2} \mathrm{O}$ ), ausencia clínica detectable de hipo o hipervolemia, Na urinario elevado $>20 \mathrm{mEq} / \mathrm{l}$ y ausencia de otras causas de hipoosmolaridad (1). El SIADH se ha descrito recientemente como efecto secundario asociado con varios fármacos ISRS, fundamentalmente con la fluoxetina responsable del $75,3 \%$ de los casos, habiéndose implicado también con menos frecuencia la paroxetina (2), sertralina (3) y la fluvoxacina (4). El síndrome inducido por estos fármacos suele aparecer pronto tras el comienzo del tratamiento, con una media de 13 días desde su inicio (intervalo de 3 a 120 días). Se produce con dosis habituales de los fármacos, sin haber correlación entre ésta y la severidad del síndrome y casi siempre (95,8\% de los casos) es reversible tras la retirada del fármaco implicado, resolviéndose a los pocos días (intervalo descrito de resolución de 2 a 28 días tras la retirada) (4). Se ha descrito la recurrencia del SIADH no sólo tras la sustitución de un fármaco ISRS por otro del mismo grupo sino también por un antidepresivo tricíclico, lo que hace que el SIADH no sea necesariamente un efecto de clase de los fármacos ISRS $(5,2,3)$. Hay una serie de factores que pueden favorecer esta complicación como son el tratamiento concomitante con diuréticos, el bajo peso corporal y fundamentalmente la edad, presentando una especial susceptibilidad las personas mayores de 65 años $(6,7)$. El mecanismo por el que los fármacos ISRS producen SIADH permanece desconocido, sin embargo considerando la heterogeneidad estructural de los cuatro fármacos de este grupo implicados, parece improbable que esté relacionado con su estructura química. Se han descrito tres posibles mecanismos responsables: el incremento de la secreción central de $\mathrm{ADH}$, potenciar el efecto endógeno de la ADH sobre la médula renal o disminuyendo el umbral homeostático para la secreción de ADH. En este sentido, los cambios fisiológicos en la homeostasis del agua en personas ancianas (deterioro de la capacidad renal para la concentración y dilución, mayor aumento en la secreción de ADH ante similares estímulos homeostáticos) pueden subyacer en la especial sensibilidad en este grupo de edad $(4,8)$.

Los ISRS están siendo utilizados cada vez con más frecuencia en personas mayores dada la frecuencia de depresión en este grupo de edad y los efectos secundarios que presentan los antidepresivos clásicos. Debido a esto, es necesario tener en cuenta esta complicación y realizar un control analítico en los tres primeros meses en pacientes que toman paroxetina y presentan deterioro de su estado general, sobre todo si son mayores o reciben tratamiento diurético.

\section{P. Álvarez Pérez, E. Rubio Nazábal', J. Marey López', S. López Facal' ${ }^{1}$, P. Rey del Corral ${ }^{1}$}

\author{
Medicina Familiar y Comunitaria. Consultorio La Torre. \\ ${ }^{\prime}$ Servicio de Neurología. Hospital Juan Canalejo. La Coruña
}

1. TYK Chan. Drug-induced síndrome of inappropriate antidiuretic hormone secretion. Drugs Aging 1997; 11: 27-44.

2. Flint AJ, Crosby J, Genik JL. Recurrent hyponatremia associated with fluoxetine and paroxetine. AM J Psychiatry 1996; 153: 134

3. Bouman WP, Johnson H, Trescoli-Serrano C, et al. Recurrent hyponatremia associated with sertralina and lofepramine. Am J Psychiatry 1997; 154: 580 .

4. Liu BA, Mittmann N, Knowles SR, et al. Hyponatremia and the syndrome of inappropriate secretion of antidiuretic hormone associated with the use of selective serotonin reuptake inhibitors: a review of spontaneous reports. CMAJ 1996; 155: 519-27.

5. Arinzon ZH, Lehman YA, Fidelman AL, et al. Delayed recurrent SIADH associated with SSRIs. Ann Pharmacother 2002; 36: 1175-7.

6. Schouten WE, Sepers JM. Hyponatremia associated with the use of a selective serotonin-reuptake inhibitor in a an older patient. Age Ageing 2001; 30: 94

7. Kazal LA, Hall DL, Miller LG, et al. Fluoxetine-Induced SIADH: a geriatric ocurrence? J Fam Pract 1993; 36: 341-3.

8. Miller M. Fluid and electrolyte balance in the elderly. Geriatrics 1987; 42: $65-76$. 


\section{Síndrome de apnea: factor de riesgo para pseudotumor cerebral}

\section{Sr. Director:}

El pseudotumor cerebral (PTC), o hipertensión intracraneal idiopática, se caracteriza por hipertensión craneal con líquido cefalorraquídeo y estudios neuroradiológicos normales, edema de papila uni o bilateral y cefalea. La incidencia anual de PTC en la población general es de 0,9 por 100.000 habitantes, siendo más frecuente en mujeres jóvenes y obesas (1). El síndrome de apnea al sueño (SAS) es un desorden respiratorio que se caracteriza por somnolencia diurna, cansancio, pérdida de memoria, ronquidos y múltiples episodios de apnea-hipoapnea durante el sueño, siendo más frecuente en varones de edad media (2). La obesidad es un factor de riesgo significativo para el PTC y el SAS. El edema de papila se produce en las situaciones en las que el flujo axoplásmico del nervio óptico está disminuido o interrumpido y es un signo clínico esencial en el diagnóstico del PTC y ha sido descrito como una de las manifestaciones oculares del SAS $(3,4)$. Se presenta un caso en el que el edema de papila unilateral fue la clave para el diagnóstico de un pseudotumor cerebral asociado a síndrome de apnea en una paciente sin clínica oftalmológica evidente.

Mujer de 45 años que acude a la consulta de oftalmología para una revisión rutinaria. La exploración fue compatible con la normalidad, salvo un edema de papila establecido en el ojo derecho. La papila del ojo izquierdo era normal. En el estudio sistémico refería cefalea de larga evolución (casi diaria), pérdida de memoria, estado depresivo por el que recibía ayuda psicológica, somnolencia en periodos de inactividad y cansancio general de varios meses de evolución. La TAC y RNM fueron normales y la punción de LCR demostró una presión de salida de 260 mm $\mathrm{H}_{2} \mathrm{O}$ con una bioquímica normal. Todos datos compatibles con PTC. Su ICM era de 32, con una circunferencia del cuello de $48 \mathrm{~cm}$. En el estudio polisomnográfico la frecuencia de apneas/hipoapneas fue de 14 por hora de sueño, diagnosticándose síndrome de apnea. Se trató con una dieta de 1.000 calorías/día y CPAP a 6 cm $\mathrm{H}_{2} \mathrm{O}$ resultando en una dramática resolución del edema de papila y de su sintomatología sistémica al mes.

El edema de papila en este paciente tiene un doble origen, por una parte la hipertensión craneal se transmite por la vaina del nervio óptico alterando el flujo axoplásmico, por otra parte en el SAS se producen múltiples episodios de apnea-hipopnea nocturnos asociados a hipoxemia/hipercapnia, resultado en un incremento de la presión intracraneal secundaria a vasodilatación cerebral, así el $70 \%$ de los pacientes con pseudotumor cerebral asocian alteraciones en el sueño y la más significativa entre ellas es el síndrome de apnea (2).

Sugerimos que el SAS puede ser un factor de riesgo de primera magnitud en el desarrollo de PTC. Estos pacientes, generalmente jóvenes, son de alto riesgo para desarrollar pérdida visual severa o incluso ceguera, pudiendo presentarse antes que el resto de los síntomas sistémicos, por eso recomendamos que a todos los pacientes con edema de papila y estudios neuroradiológicos normales se les descarte un pseudotumor cerebral y se les pregunte sobre síntomas de apnea e igualmente, a todos los pacientes con el diagnóstico de PTC y/o SAS se les complemente con un estudio oftalmológico.

\section{M. Asensio Sánchez, J. Merino Angulo ${ }^{1}$, A. Narros Giménez ${ }^{1}$}

Servicios de Oftalmología y ${ }^{1}$ Medicina Interna. Hospital General del Servicio Castellano-Leonés de Salud. Medina del Campo. Valladolid

1. Kesler A, Goldhammer Y, Gadoth N. Do Men with pseudotumor cerebri share the same characteristics as women? A retrospective review of 141 cases. J Neuroopthalmol 2001; 21: 15-7.

2. Marcus DM, Lynn J, Miller JJ, Chaudhary O, Thomas D, Chaudhary B. Sleep disorders: a risk factor for pseudotumor cerebri? J Neuroopthalmol 2001; 21: 121-3

3. Wolin MJ, Brannon WL. Disk edema in an overweight woman. Surv Ophthalmol 1995; 39: 307-14.

4. Purvin VA, Kawasaki A, Yee RD. Papilledema and obstructive sleep apnea syndrome. Arch Ophthalmol 2000; 118: 1626-30. 